\title{
Implementation of Support Vector Machine for Identification of Skin Cancer
}

\author{
Neela A G \\ Assistant Professor, Department of Electronics and Communication Engineering JSS Academy of Technical \\ Education, Bangalore, Karnataka, India
}

Received: 21 September 2019; Accepted: 15 October 2019; Published: 08 November 2019

\begin{abstract}
Skin cancer is one of the most death causing cancer with the increase of infections on skin due to various parameters of the nature, atmosphere and geographical area .The abnormal growth of skin cells has become common in today's world this abnormal growth is termed as skin cancer. Skin cancer mostly develops on the part of the skin which is exposed to sun light continuously or for long duration develops on body exposed to sun light, but it can occur anywhere on the body. Skin cancer in beginning stage is curable. Patient's life can be saving from skin cancer by early \& fast detection. Early detection of skin cancer in achievable at beginning stage with the new technology. Biopsy method was used to detect the cancer in the earlier days. During biopsy, a small part of the skin tissue is extracted from the carcinoma patient; this part of the tissue will be processed in various laboratories for the identification of the presence of infected cells and the stage at which the cancer is in. Biopsy was a very time consuming and painful for the patients, and the result of biopsy process was not accurate and correct. To overcome the loner procedure and to increase the accuracy Support Vector Machine Algorithm was used in identifying the infection/ Carcinoma at the early stage and cure the infection before it leads to death.

Index Terms: Carcinoma, Dermoscopy, ANN, GLCM, SVM, Image Processing, Malignant Basal Cell Carcinoma (BCC), Squamous Cell Carcinoma (SCC).

(C) 2019 Published by MECS Publisher. Selection and/or peer review under responsibility of the Research Association of Mode rn Education and Computer Science
\end{abstract}

* Corresponding author.

E-mail address: neela_guruswamy@jssateb.ac.in 


\section{Introduction}

Cancer The uncontrolled growth of the infected cells influencing the infection to the neighboring cells leads to cancer this is known as skin cancer. The rapid multiplication of the malignant cells leads to tumor. The malignant tumors are formed due to spreading of skin cells rapidly. Human Skin consists of 3 layers the outermost layer of skin known as epidermis. After epidermis the net layer below it is known as dermis it contains hair follicles, sweat glands and connective tissues.

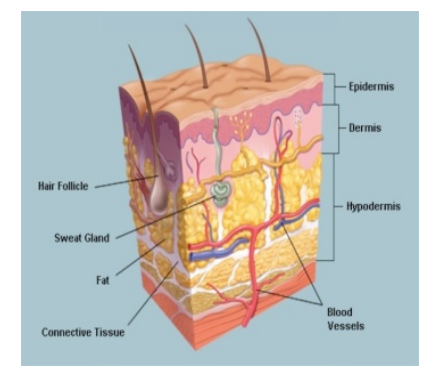

Fig 1. Skin Picture

The final and the last deep tissues is known as hypodermis it is made of fat and connective tissues.

Skin cancer is further divided into melanoma and non melanoma cancer. When unrepaired DNA damages to skin cells there is development of the cancer which is called as melanoma skin cancer, this cancer develops due to the exposure to the sunlight and UV radiations and the mutation of this type of cancer occurs very fast and can also occur due to the genetic effects. As we know there are pigment producing layer on the skin this type of cancer arises in the layer of the skin where production of pigment takes place. The resembles of this cancer is similar to moles. The color of melanomas are brown in color or black in color not only they restrict the color to brown and black they can also sometime turn out to be in pink, red, purple, white ,blue and in color the shade of the skin. Hence it is very dangerous to expose the parts of the skin to the UV rays which effect severely to the skin and causes the sunburn and in turn the cancer and this can be common to those people who are genetically predisposed to the disease.

Non-Melanoma skin cancer is Basal Cell Carcinoma which develops into the deeper layer of the skin compared to SCC and these cancers are mostly seen on the head, face and neck. Squamous cell carcinoma is the 2nd most common type of skin cancer which infects the human these days these starts in the squamous cells which are most widely found on the epidermis and can multiply faster than the BCC these are the two major types of skin cancer which infects the humans these days and the other cancer which is less seen its effect is known as malignant carcinoma though the cancer is less seen but once this infects the cells it multiplies very fast and rapidly, hence identification and curing of this cancer is more important.

Hence identification and curing of the skin cancer in the earlier stages can help in saving the life of a person. In UK the almost 75 in 100 people suffering from skin cancer are infected with BCC. The detection of this cancer at the early stage can completely be cured whereas still $0.1 \%$ of the cases may be left out where the early detection also cannot be cured as the level the cancer has spread to the skin may be into the deeper region of the skin. There are chances of the skin cancer to come back on the same infected place even after curing this is known as reoccurrence of the cancer.

Compared to BCC, SCC can be cured completely as it spreads on the outer part of the skin hence if identified at the right time the curing can be such that there is no scar of carcinoma infection at all and these 
carcinoma are late in there multiplication but if not recognized for the longer duration they can become active and spread to the nearby cells and can be more dangerous than BCC.

1.In case if the diagnosis was incorrect there was the possibility of spreading of diseases to other parts of patient body before treatment.

2.To overcome all the obstructers in the detection the Support vector Machine is proposed this is more effective and efficient.

3.Initial detection of the skin cancer is through the image processing technique to get the clear and sharp images any application of the external oil on the skin is not necessary so this method of detection of cancer on the skin using image processing and SVM is clear, clean and fast technique.

\section{Literature Review}

There are various activities and algorithms proposed related to the skin cancer and its curing. Detection of Skin cancer was based on maximum entropy threshold technique where the Gray Level Co-occurrence Matrix (GLCM) along with ANN was used for classification of the images with back propagation technique [1].

The classification of $\mathrm{K}$ clustering $(\mathrm{KNN})$ technique was used and was not able to achieve the desired rate of and various data pre processing methods are used in diagnosing the disease and maximum frequent set algorithm adds to this in order to classify the images [6].

The other techniques used are segment the clustering technique in this the features can be considered and taken from the image using asymmetry index border color index diameter technique [11].

Rules based forward chaining approach technique is used to detect and cure the skin cancer in this paper the author discusses about the skin diseases which are affecting the kids and he discusses about the various algorithms which are used for classification. Like Ada Boost, Bayes Net, MLP and Naïve Bayes to identify and cure the carcinoma. The drawback of this paper was it could only work with the infections of Eczema, Impetigo and Melanoma [8].

Melanoma diagnosis applied on a set of digital images. Features extracted by using gray level Co-occurrence matrix along with classifier such as Multilayer perception to segregate between malignant and non malignant images [9].

\section{METHOD \& METHODOLOGY}

In this paper a automatic Skin cancer detection system is used to identify the malignant cells and to do this a algorithm is built known as support vector machine algorithm which is used for classification and regression analysis and the extraction of the features required for processing is done using GLCM.

\section{BLOCK DIAGRAM}

There are various features that are not detectable by naked eye to find these there are various dermoscopy techniques such as solar scan, ELM, XLM, TLM.

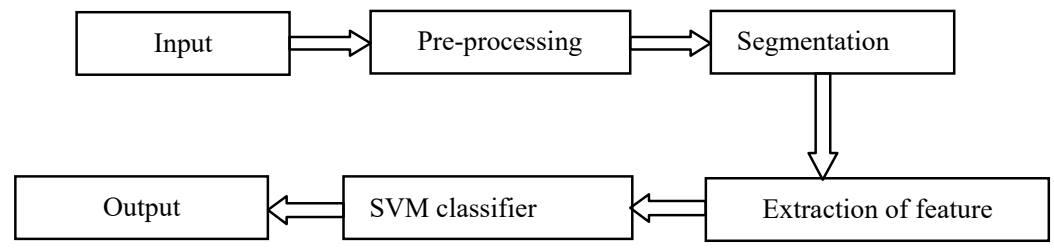

Fig 2. Block Diagram 


\section{Extraction of the required feature}

The biological important features are extracted from the image obtained from the dermatoscope device. The image captured by the device are not proper in nature these images are known as raw image $t$ extract the required and desired features from these a lot of process has to be undergone this process are pre defined as image processing techniques.

\section{Input image}

The raw images captured from an hand help instrument know as dermatoscope acts as the input the system which segregates the malignant and benign cells and tissues. The devices acts as a magnifier which is used to capture the image from any parts of the body which is infected without altering the actual features of the image Fig 3 shows the image of the device used and Fig 4 is the captured raw image.

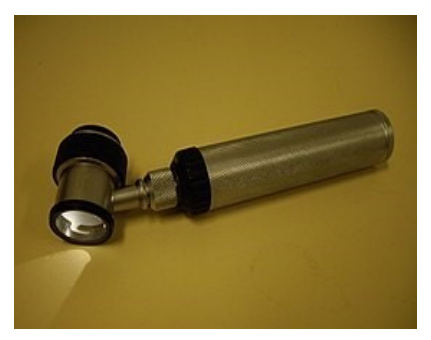

Fig 3. Dermatoscope Device

Fig 4. Captured raw image

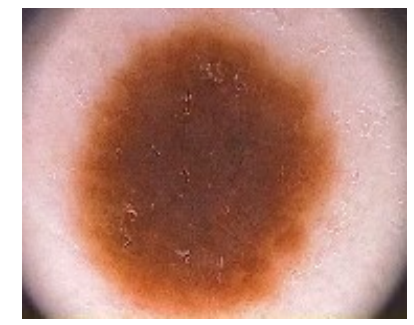

\section{Image pre-processing}

The image obtained from the scanning process has to undergo various pre processing stages to remove the unwanted details and the noise and pre processing stages also help to enhance the required features for diagnosing and detection. Using good pre-processing technique means better accuracy. The image preprocessing also involves a stage where the image pixel are enhanced to identify the region of interest.

Image pre-processing stage involves following process, that is: Image enhancement:

Image enhancement technique is a process where the each and every pixel of the image is enhanced to the required level where the infection is clearly identified Fig 5 shows the enhanced image. 


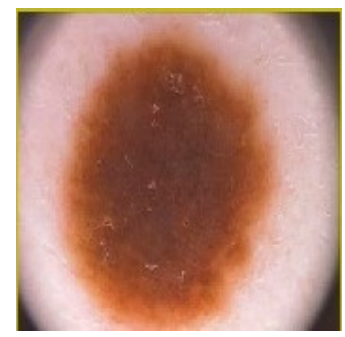

Fig 5. Enhanced image

\section{Gray scale conversion}

Gray scale image contains only intensity information. The value of each pixel is a single sample, representing only quantity of light. The conversion to grayscale makes the identification and classification quick and effortless than the RGB image. The measurement of grayscale is only through brightness this brightness is in the range of 0 to 255 where the 0 refers to black and the other end 255 refers to white [4]. During the conversion of RGB to gray scale image following equation is used Grayscale intensity $=0.299 \mathrm{R}+$ $0.587 \mathrm{G}+0.114$ B Fig 6 shows the gray converted image.

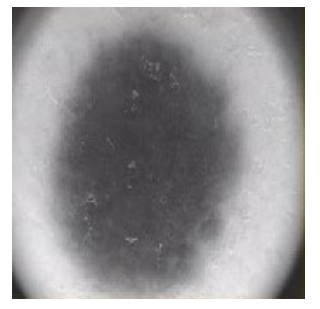

Fig 6. grey converted image

\section{Noise removal and Binarization}

The grey concerted image has to be digitized in order to know the region of interest accurately and clearly as there should be no mistake of identifying the carcinoma hence binarization plays a important role in image processing and the noise removal also should be done with the at most care as noise itself says it's as an unwanted feature in the image Fig 7: Binarization of Image

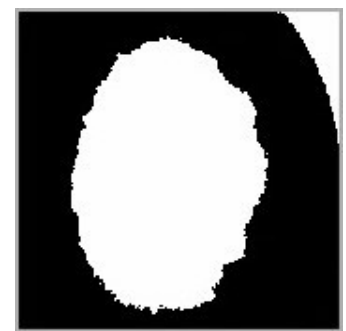

Fig 7. Binarization of Image Segmentation 
Region of interest is removed in segmentation. Each pixel in that region is having similar attributes. Otsu's thresholding algorithms used in segmentation [5]. To segment (separate ) the region of interest the foreground and the background of the gray scaled image has to be differentiated the histogram of the original image is calculated and used to do this as its in terms of binary transformation to calculate the histogram maximum entropy method is applied Fig 8 shows the segmented image.

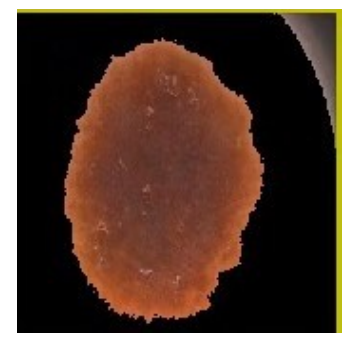

Fig 8. Segmented image Feature extraction

To extract the required feature of interest from the whole scanned and processed image feature extraction technique is used. GLCM algorithm is used for feature extraction.

\section{GLCM}

Gary Level co-occurrence matrix is used to create the GLCM algorithm this works on the process let i be the intensity of the pixel and $\mathrm{j}$ be the neighboring of the pixel the co occurrence matrix calculates the relationship between the $I$ and $j$ that is how often the parameter $i$ occurs with respect to $j$ and this is related as $(\mathrm{i}, \mathrm{j})$ this can be said that as a relation between the interested pixel to that of the neighboring or adjacent pixel hence the resultant GLCM is simply the sum of the number of times that the pixel with value i occurred in the specified spatial relationship to a pixel with value $\mathrm{j}$ in the input image.

The implementation of the GLCM takes place by increasing the size of the image. GLCM reduces the intensity of the pixels from the value 256 to 8 . The number of grey level of the image determines the GLCM size .using num levels in the GLCM the gray levels of the pixel images can be controlled and the gray levels can be limited.

- Mean

$$
\mu=\frac{1}{N} \sum_{i=0}^{N-1} x_{i}
$$

- Standard deviation

$$
\sigma^{2}=\frac{1}{N-1} \sum_{i=0}^{N-1}\left(x_{i}-\mu\right)^{2}
$$


- Entropy

$$
\sum_{i, j=0}^{N-1} x \ln \left(P_{i j}\right) P_{i j}
$$

- RMS

$$
x_{R M S}=\frac{1}{N} \sum_{n=1}^{N}\left|x_{n}\right|^{2}
$$

\section{Classifier}

The classification cost can be made minimum in the usage of SVM classifiers .by choosing a proper kernel the SVM is made advantageous as the framework defined will be of uniform. There are training and testing phase involved in the classification which is as shown in Fig 9 and Fig 10 shows the classified image after the application of SVM.

The process involves two phases namely training phase and testing phase. In training the phase the collected samples are tested for the malignancy and labeled with the names. In testing phase the new samples as and when they arrive is tested using SVM algorithm for the malignant features and comparison is done based on the passing of the test they are segregated into malignant and benign cells.

The SVM classifier is applied on the statistical texture features to predict the malignancy of the skin lesion. Each skin image in test set is classified by comparing it against the skin images in the training set. The training set consists of both normal and cancer skin images and skin disease images. The comparison is performed using the local features obtained in the previous step. Compared to other classifiers SVM can be more advantageous.

Feature extraction stage the input raw image is tested under various conditions and send to the database in order to match the other input for the similarity and then classify them into the categories.

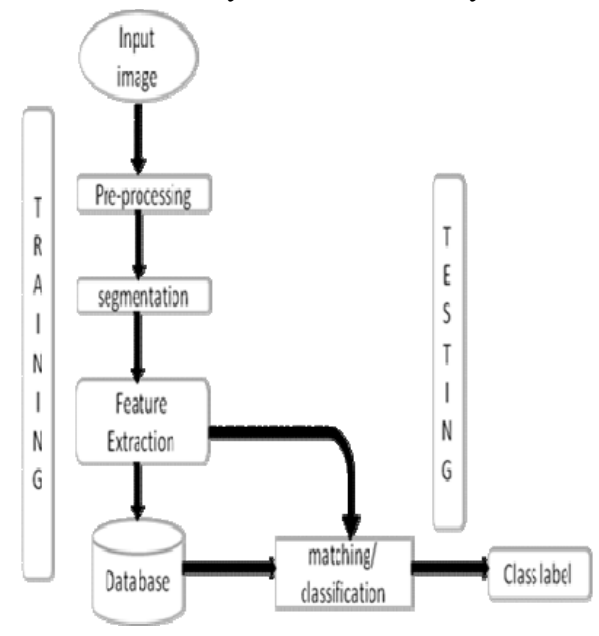

Fig 9: Testing and Training Flow 


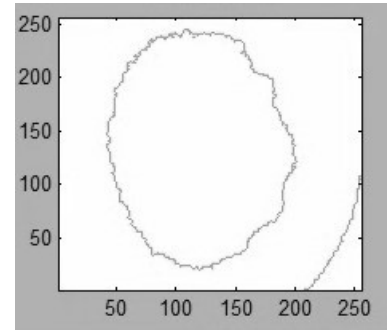

Fig 10: Classified image after the application of SVM

\section{Result}

Skin Cancer images are collected from cancer hospitals data set .These images have undergone through image processing techniques. GLCM algorithm is used to summarize the structure of the image comparing the features of the neighboring pixels. Table 1 and Fig 11 show the implementation of the algorithm.

Table 1: Comparison parameters of the images

\begin{tabular}{|c|c|c|}
\hline FEATURES & NON MALIGNANT & MALIGNA NT \\
\hline MEAN & 0.0238 & 0.0835 \\
\hline STD-DEVIATION & 0.1523 & 0.2767 \\
\hline ENTROPY & 0.1620 & 0.4144 \\
\hline RMS & 0.0542 & 0.1645 \\
\hline VARIANCE & 0.0190 & 0.0612 \\
\hline SMOOTHNESS & 0.9993 & 0.9998 \\
\hline
\end{tabular}

\section{Conclusion}

At the end it can be understood that the proposed system of skin cancer detection is implemented using GLCM and SVM to classify whether the input image affected by disease or not. Accuracy is more. It is painless and consumes less time than biopsy method. It is more useful for both patient as well as doctor. This project can be further enhanced with KNN and other algorithms were the accuracy can be increased and using more data can also increase the accuracy rate of detection. 


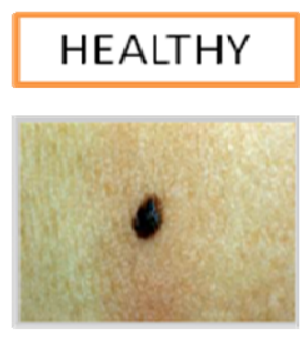

\section{UNHEALTHY}
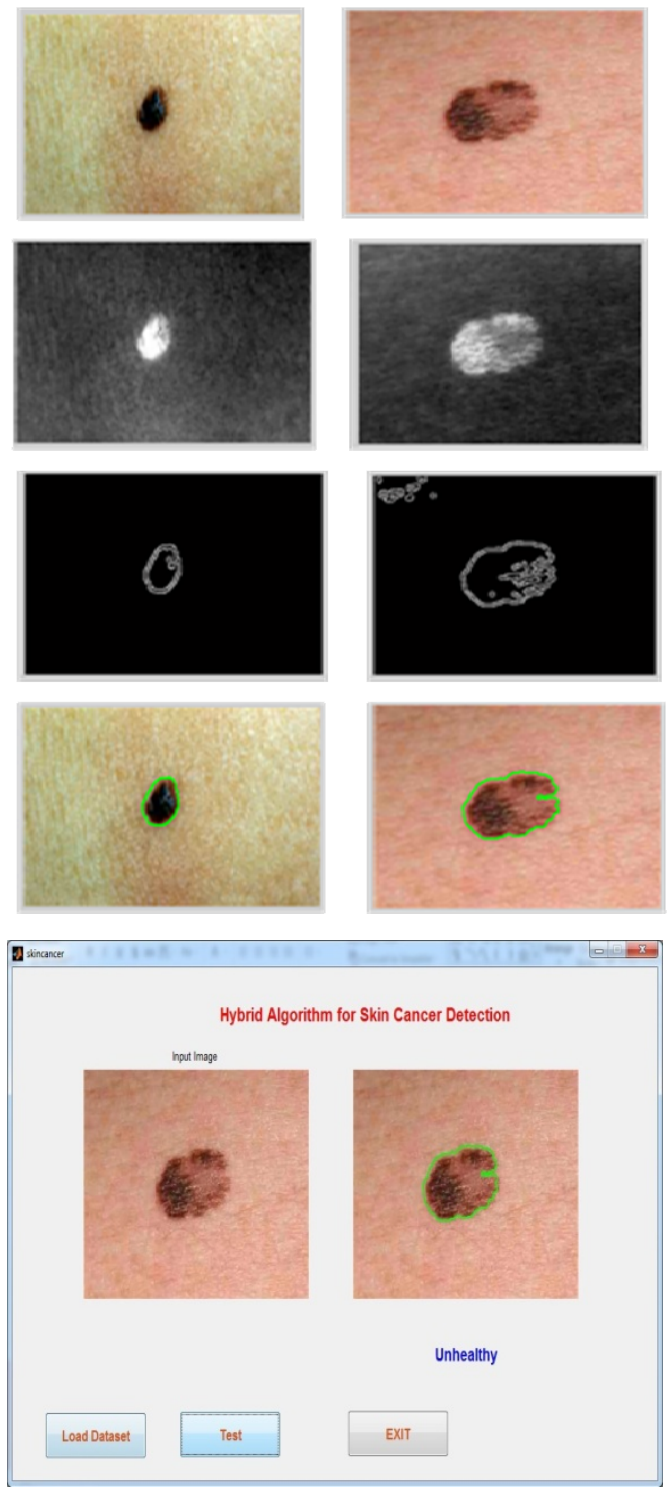

Fig 11: final output of the image 


\section{ACKNOWLEDGMENT}

I would like to thank my parents and my family who has helped me to carry out the work successfully. I thank my guide and my colleagues at the institute for their support.

\section{References}

[1] C.Nageswara Rao, S.SreehariSastry and K.B.Mahalakshmi "Co-Occurrence Matrix and Its Statistical Features an Approach for Identification of Phase Transitions of Mesogens", International Journal of Innovative Research in Engineering and Technology, Vol. 2, Issue 9, September 2013.

[2] Santosh Achakanalli\& G. Sadashivappa ," Statistical Analysis of Skin Cancer Image -A Case Study “, International Journal of Electronics and Communication Engineering (IJECE), Vol. 3, Issue 3, May 2014.

[3] "Digital image processing" by jayaraman. Page 244,254-247,270-273. (Gray level, median filter).

[4] Algorithm For Image Processing And Computer Vision .Page 142-145. (Thresholding)

[5] Kawsar Ahmed, TasnubaJesmin, "Early Prevention and Detection of Skin Cancer Risk using Data Mining", International Journal of Computer Applications, Volume 62- No.4, January 2013.

[6] Maurya R, Surya K.S,"GLCM and Multi Class Support Vector Machine based Automated Skin Cancer Classification, "IEEE journal, vol 12, 2014.

[7] A.A.L.C. Amarathunga," Expert System for Diagnosis of Skin Diseases", International Journal of Scientific \& Technology Research, Volume 4, Issue 01, 2015.

[8] Mariam A.Sheha,"Automatic Detection of Melanoma Skin Cancer", International Journal of Computer Applications, 2012.

[9] Anshubharadwaj, "Support Vector Machine", Indian Agriculture Statistics Research Institute.

[10] M.Chaithanya Krishna, S.Ranganayakulu, "Skin Cancer Detection and Feature Extraction through Clustering Technique", International Journal of Innovative Research in Computer and Communication Engineering, Vol. 4, Issue 3, March 2016.

[11] Dr.J.Abdul Jaleel ,Sibi Salim and Aswin R.B. “Artificial Neural Network Based Detection of Skin

[12] Cancer", International Journal of Advanced Research in Electrical, Electronics and Instrumentation Engineering,Vol. 1, Issue 3, September 2012.

[13] Anal Kumar Mittra and Dr.Ranjan Parekh, “Automated Detection of Skin Diseases" International Journal of Engineering Science and Technology (IJEST) Vol. 3 No. 6 June 2011.

[14] Catarina Barata, Margarida Ruela , Mariana Francisco, Teresa Mendonca and Jorge S. Marques, "Two Systems for the Detection of Melanomas in Dermoscopy Images Using Texture and Color Features", IEEE SYSTEMS JOURNAL 2013.

[15] Md.Amran Hossen Bhuiyan, Ibrahim Azad, Md.Kamal Uddin, Image Processing for Skin Cancer Features Extraction, International Journal of Scientific and Engineering Research Volume 4, Issue 2, ISSN 2229-5518, February-2013.

[16] A.Aswini, E.Cirimala, R.Ezhilarasi, M.Jayapratha, Non Invasive Screening and Discrimination of Skin Images For Early Melanoma Detection, International Journal of scientific research and management (IJSRM), Volume, 2, Issue, 4, Pages 781- 786, 2013 
[17] Arati P. Chavan D. K. Kamat Dr. P. M. Patil, CLASSIFICATION OF SKIN CANCERS USING IMAGE PROCESSING, International Journal of Advance Research in Electronics, Electrical Computer Science Applications of Engineering Technology Volume 2, Issue 3, , PP 378-384 June 2014.

[18] Chaithanya Krishna, S.Ranganayakulu, "Skin Cancer Detection and Feature Extraction through Clustering Technique", International Journal of Innovative Research in Computer and Communication Engineering, Vol. 4, Issue 3, March 2016.

[19] J Abdul Jaleel, Sibi Salim, Aswin.R.B," Computer Aided Detection 01 Skin Cancer", International Conference on Circuits, Power and Computing Technologies, 2013.

\section{Author's Profile}

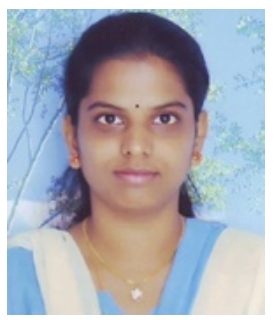

Mrs. Neela A G , Assistant Professor of JSS Academy of Technical Education, Bangalore and Research Scholar of JSS Science and Technological university, Mysore. Life time member of ISTE, and have published 3 international journals and have attended 2 conferences, guided UG students in there smooth conduction of the projects.

How to cite this paper: Neela A G. "Implementation of Support Vector Machine for Identification of Skin Cancer", International Journal of Engineering and Manufacturing(IJEM), Vol.9, No.6, pp.42-52, 2019. DOI: 10.5815/ijem.2019.06.04 